\title{
Privacy and Socially Assistive Robots - A Meta Study
}

\author{
Tanja Heuer and Ina Schiering and Reinhard Gerndt \\ Ostfalia University of Applied Sciences \\ Wolfenbüttel, Germany \\ \{ta.heuer, i.schiering, r.gerndt\}@ostfalia.de
}

\begin{abstract}
This paper investigates studies about socially assistive robotics with focus on privacy and ethical concerns. Therefore, the privacy aspects are considered and the concerns expressed by users with regard to privacy are examined additionally. It becomes clear, there are still a lot of concerns regarding the use of robots, that's why robots are not well accepted so far. To get a more transparent view on that, two models are introduced which might improve the understanding towards important privacy aspects.
\end{abstract}

Keywords: socially assistive robot, Robot Ethics, HRI, Human-Robot Interaction, Human-Robot Friendship, Machine Ethics, Privacy

\section{Introduction}

Some years ago robots were exclusively present in research laboratories and industrial facilities. Because of a miniaturization of components and the decline in price, robots are increasingly used for personal applications as e.g. smart home applications and social interaction now. According to the International Federation of Robotics (IFR) "about 5.4 million service robots for personal and domestic use were sold [in 2015], 16\% more than in 2014." [24]. Two thirds of those "are robots for domestic tasks, including vacuum cleaning, lawn-mowing, window cleaning and other types" [24] and it is estimated that these numbers will rise up to 30 million until 2019. "Sales of robots for elderly and handicap assistance will be about 37,500 units in the period of 2016-2019. This market is expected to increase substantially within the next 20 years" [24].

Tasks of domestic robots (for elder care) are mainly categorized into three areas [46]: "(1) to assist the elder[s], and/or their carers in daily tasks; (2) to help monitor their behavior and health; and (3) to provide companionship". The aim of social robots is a (semi-) autonomously interaction with humans in a respectful way [35]. Several of the intended domestic tasks require sensors as e.g. cameras or microphones to allow individualized user interactions. Users of domestic robots expect a conversation in a "natural human-like manner" [19]. Because of these user expectations, robots are often considered as living beings like humans or animals and are treated as friends that accompany humans in their private environment [21]. Robots will be dangerously ubiquitous, but, as for 
other smart devices as e.g. smart phones, wearables, etc. that accompany users in their daily life, the privacy risks are not obvious. Hence, privacy should be an important aspect in robotics, but is not taken into consideration in a satisfactory way yet. At the moment robots are designed from an engineering perspective with a main focus on functionality. Privacy and other ethical aspects of robotics are investigated in general, but not adequately integrated in the engineering process of robotics.

In this paper the role of privacy in "Robotic Ethics" is investigated in the context of user studies with assistive robots. There the perception concerning privacy of different user groups is investigated and the effect of the type of robot on the perception of privacy is examined. Approaches to make privacy risks more transparent are discussed as a first step to foster the consideration of privacy in robot design processes.

The considered so called Socially Assistive Robots (SAR) together with an approach for categorization are described in section 3. An overview of ethical concerns towards robotic is presented in section 4 . The role of privacy in this ethical context is emphasized. In section 5 an overview about Socially Assistive Robots is given and privacy concerns of users are stated and evaluated in section 6 followed by discussion and conclusion.

\section{Methodology}

The basis of the meta study is a literature survey. The retrieval was based on queries in databases and search engines for scientific publications, i.e Google Scholar and ScienceDirect. In addition, the following relevant conferences were considered ISCR (International Conference on Social Robotics), ISRR (International Symposium on Robotics Research) and RO-MAN (Robot and Human Interactive Communication). Main key words as "social robot", "robot/robotic and privacy", "robot/robotic study" and "robot/robotic and ethical aspects" were used. In addition, other surveys as Leite et al. [36] who already gave an overview of social robots for long-term interactions, are considered. Because of an increasing use of robots for home and health care the collection of papers has been limited to the last $\sim 10$ years.

Investigated robots are sorted into categories explained in section 3 . The categorization which is adopted by Fong et al. [18] points out the different types of robots and their appearances. Based on this categorization, it should be examined whether the perception of privacy depends on the appearance or on specific functionalities. Further we want to investigate, how participants and users perceive privacy issues and how users express their concerns in the context of the studies.

For each paper the duration, target group and the number of participants is described and summarized in Figure 5. Duration gives an overview of the length of the studies. If a study for example just lasts one hour, the user may not think about privacy risks of the investigated robot. Also the target group is important for the assessment of human-robot interaction (HRI), since children 
might interact in a different way with robots than older people. The number of participants is also important. Studies with a larger number of participants could achieve more significant results about the perception of a robot.

The main focus in this meta study is on the ethical, especially privacy concerns, which are mentioned by the users during the HRI studies. With the introduction of individualized conversation between robots and users, the utilization of sensors, e.g. cameras and microphones in addition to standard sensors for autonomous behavior as ultrasonic sensors or laser range scanners, increases. But even though sensors use is increasing, it is not transparent to the user which data is recorded, stored and transmitted. In the studies users express skepticism, interest and curiosity. It is interesting to investigate, which of these attitudes prevails.

\section{Socially Assistive Robots}

In the context of this study social robots are in the focus. A robot is called social, when it is able to react to human actions in a (semi-) autonomously way either by speech or movements. Movement in this sense includes moving only the head or another part of the robot as an arm or a leg. The area of socially assistive robots (SAR) is defined as "a class of robots that is the intersection of assistive robotics [...] and socially interactive robotics [...]" [14]. Hence, socially assistive robots are able to substantially support human beings in many areas of their daily life: companionship, entertainment, security, transportation, education, customer service, personal assistance, sales and tourist guidance [46]. A huge variety of robots are developed to realize one or more of these tasks. Robots in this sense are divided into four categories defined by Fong et al. [18] as described in the following.

Functional The focus of this category is the practical benefit of the robots. These robots are designed with the focus on the intended use. For instance, requirements in health care like a storage space or a removable tray need to be taken into account for the construction [39]. As an example the robot Pillo serves as a reminder of medication (see Fig. 1). It is able to store medicine and to dispense the medicine depending on the needs of the user [1]. Therefore it has a closed compartment for the medicine with restricted access and a special place is designated for a glass of water. Although it is designed in an abstract functional way, it shows eyes on its screen to look sympathetic.

Caricatured Cartoons and caricatures are the background of this category. In cartoons, for example, characteristic features of a creature are particularly emphasized. By highlighting these features, other elements are neglected. The attention of the user should be focused on the emphasized part. Scheeff et al. used cartooning techniques to design the robot Sparky [43]. This robot looks like a small turtle with focus on the face and the facial expressions. Another example 


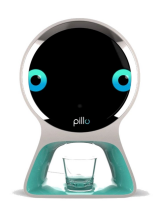

Fig. 1. Pillo ${ }^{1}$

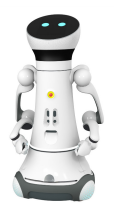

Fig. 2. Care-O-bot $4^{2}$

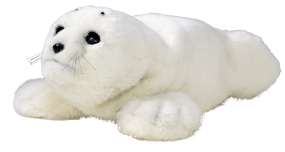

Fig. 3. Paro ${ }^{3}$

is the Care-O-bot 4 (see Fig. 2). It is equipped with arms for fetch-and-carry tasks and wheels instead of legs for moving. The head is a touch screen and it represents a face by showing abstract eyes as the Pillo.

Zoomorphic A third design of robots is the imitation of zoomorphic creatures. There the robot design is either based on realistic or fantasy animals. In the context of children and elder care animal-like creatures are often used, eg. Paro and Aibo for entertainment. Fong et al. [18] suggest that for humans it is easier to build relationships to animals and interactions are possible on a lower level. These robots often do not have a wide range of functionalities but mainly act as a companion. Paro, a seal robot is able to move its head, tail and the eyes when it is touched (see Fig. 3). Additionally it is able to express a sense of well-being by sounds.

Anthropomorphic Anthropomorphism signifies the attribution of human appearances, behaviors and other characteristics to non-human-objects. The aim of anthropomorphic robots is to make human like actions of a robot easier to understand for human beings [13]. A meaningful human-robot interaction in a social way is supposedly easier when the robot has a physical and a mental personification [6,42]. DiSalvo et al. [11] suggest "that interaction through speech and movement will greatly effect the perception of humanness in robots". With the humanness of a robot, other deficits can be covered up.

\section{Ethical Concerns in SAR and the Role of Privacy}

In this section different concepts of ethical concerns in SAR are introduced. In 1942 Isaac Asimov created the first general three laws of robotics stating that a robot must obey a human being and is not allowed to hurt human beings. With increasing interest in the use of robots in various application areas, these rules were further specified in 2009 [38]. These two approaches comprise mainly

\footnotetext{
${ }^{1}$ https://assets.entrepreneur.com/content/3x2/1300/20160630152708-pillo.jpeg

2 http://www.care-o-bot-4.de/

${ }^{3}$ https://www.thestar.com/life/breakingthrough/2014/06/09/robot_gets_seal_of_ approval.html
} 


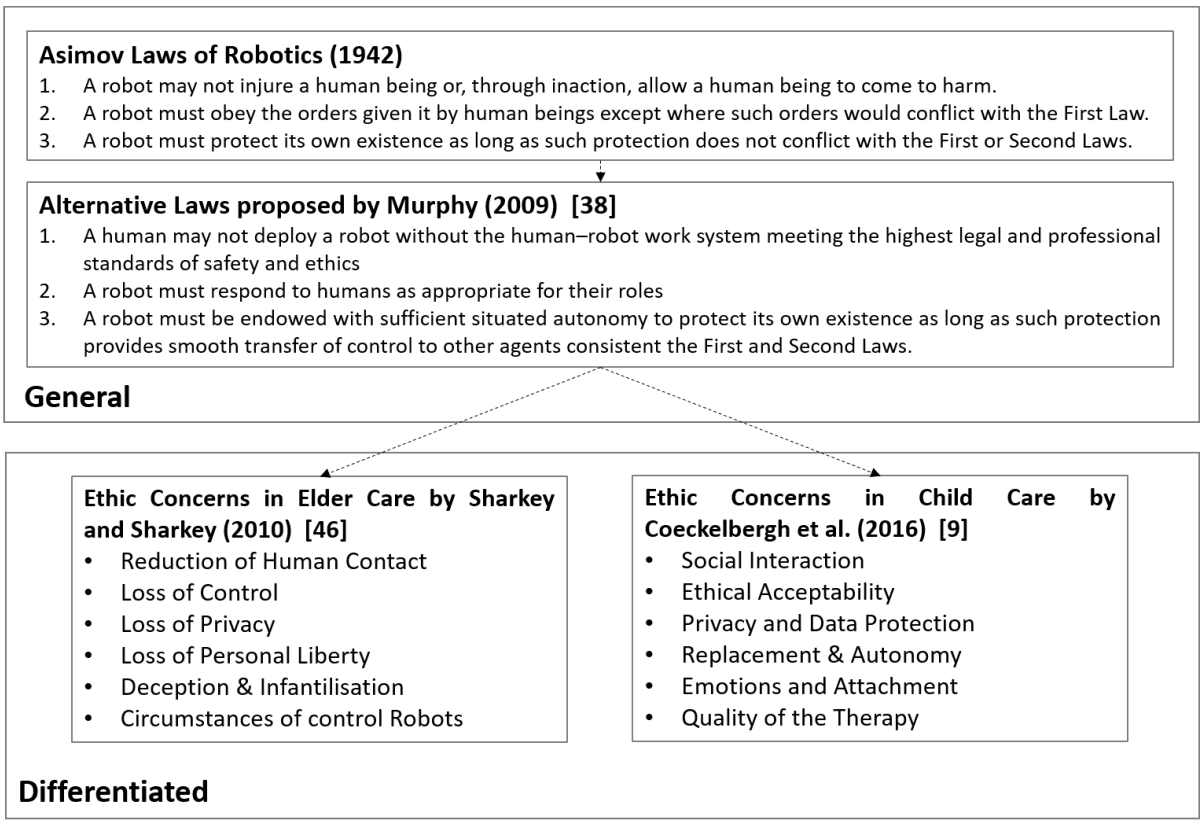

Fig. 4. Overview of General Robotic Laws and Ethical Concerns

a professional standard for a safe human-robot interaction but specific ethical aspects of this safe interaction are not considered. A detailed overview of ethical concerns is proposed by Sharkey \& Sharkey [46] and Coeckelbergh et al. [9] focused on specific topics in health care. Whereas Sharkey \& Sharkey [46] discuss concerns of using robots in elder care, Coeckelbergh et al. [9] investigate ethical concerns of using robots in therapeutic childcare (see Fig. 4).

These detailed approaches show in general a broad consensus. Aspects like loss of privacy and data protection are mentioned. Also the ethical acceptability towards robots is introduced [9]. Several of these ethical concerns comprise also privacy aspects. For example, the personal liberty and the self-control of personal thinking and acting needs to be guaranteed. If the robot is regarded as a friend, emotions and attachment play a role. A friend is more entrusted than a machine. Therefore this needs to be investigated in a more detailed way.

Denning et al. [10] developed a questionnaire of considerable fundamental secure and privacy-respecting issues for the development of household robots. They focused on network security and possible attacks, e.g. spying on homes, acquisition of login credentials. The questions are divided into Social, Environmental, Technical Questions and Security and Privacy Questions (see Table 1). 


\begin{tabular}{|c|c|}
\hline $\begin{array}{l}\text { Social, Environmental, and } \\
\text { Technical Questions }\end{array}$ & Security and Privacy Questions \\
\hline $\begin{array}{l}\text { What is the intended function of the } \\
\text { robot? }\end{array}$ & $\begin{array}{l}\text { Does the robot create new or amplify } \\
\text { existing privacy vulnerabilities? }\end{array}$ \\
\hline How mobile is the robot? & Does the robot create new or amplify \\
\hline What actuators does the robot possess? & existing physical integrity vulnerabilities? \\
\hline What sensors does the robot possess? & Does the robot create new or amplify \\
\hline $\begin{array}{l}\text { What communication protocols does the } \\
\text { robot support? }\end{array}$ & $\begin{array}{l}\text { existing physical safety vulnerabilities? } \\
\text { Does the robot create new or amplify }\end{array}$ \\
\hline Who are the intended users of the robot? & existing psychological vulnerabilities? \\
\hline $\begin{array}{c}\text { What is the robots intended operational } \\
\text { environment? }\end{array}$ & $\begin{array}{l}\text { Can the robot be combined with other } \\
\text { robots or technologies to facilitate an }\end{array}$ \\
\hline $\begin{array}{l}\text { Besides the intended users of the robot, } \\
\text { what other people (and animals) will be } \\
\text { in the the robots environment? }\end{array}$ & attack? \\
\hline $\begin{array}{l}\text { What kind of development processes are } \\
\text { in place? }\end{array}$ & \\
\hline
\end{tabular}

\section{$5 \quad$ Studies about (Socially) Assistive Robots}

This section describes studies about assistive robots of the last decade with a focus on ethical concerns and privacy aspects mentioned by the participants. To answer the questions about user groups, the overview of the studies is divided into categories shown in Figure 5 on the $\mathrm{x}$-axis. Participants are divided into children, families, older people and mixed participant groups. The y-axis lists the different types of robots investigated. The duration and the number of participants of the studies is represented by icons. If a robot is used for a longer time by the participants, more interaction is possible and the user is able to build up a personal attitude towards the robot. The number of participants is an important indication of the significance of the study.

\section{$5.1 \quad$ Functional}

Families: Studies with the vacuum cleaner Roomba are all carried out in family environments. Families comprise in this context single households, households with children or couples. These studies were conducted in 2007 [4], 2009 [48], 2011 [16] and 2013 [15] and evaluated the change of cleaning routines and activities over time. Ethical concerns are mentioned by Fink et al.[15] stating "When people did not trust/rely on the robot, they did not want to leave the room/home when the robot was switched on". Even if the robot has no camera or microphone "[...] the Roomba changed people's cleaning activities [...]" [4]. 
Older People: Beer et al. [3] conducted a study on older people using the MRP (mobile remote telepresence system). Twelve older adults took part in this study which consisted of two sessions. The goal was the improvement of social communication with other people. The robot Cafero is used in a residential home by residents and staff for about 30 minutes [7]. It provides medication management based on web services and uses a camera for face recognition. There are no concerns mentioned and the researchers ensured the confidentiality of the data and employed anonymization techniques. In 2016 the PeopleBot was used and contrasted to Care-o-Bot in a study at the University of Hertfordshire on older people [49]. The robot e.g. offers functionalities as video telephony and the participants were allowed to use the robots for whatever they want to and whenever they like.

Mixed: Other studies also at the University of Hertfordshire [30, 31, 50] explored the habituation effects of different participant groups working with the PeopleBot. Within the five weeks the preferences of the participants changed because they got used to the robot. Another important aspect is the fear of the loss of control mentioned by participants because of the autonomous behavior of PeopleBot [30]. In a further investigation the researchers focused on attitudes towards privacy. The participants attended a session where the concerns of using personal robots were in the focus. As main issues the storage of personal information and which data is stored are mentioned. Other concerns regarding usability and applicability are stated [50]. Kidd et al. [28] introduced a robot called Autom for helping people to reduce their weight. A camera is included for face recognition and the conversation between robot and the human is personalized based on prior usage. The ACE (Autonomous City Explorer) [55] was driving through Munich, Germany for one day asking persons for the right way. Equipped with a stereo vision camera, a touch monitor and a loudspeaker it interacted with passers-by. No ethical aspects were mentioned. A study with Spunik [32] evaluated the differences of HRI with an avatar (i.e. virtual representation of a robot on a screen) or a physical robot. Participants expressed privacy as one of their main ethical concerns during that study. To work effectively as assistant, the robot needs a lot of knowledge about the user. Another important aspect is the "damage of the privacy space", when the robot is able to move on its own.

\subsection{Caricatured}

Children: RoboVie is used in different studies of Kanda et al. [27, 26]. RoboVie acts for example as teacher for children in Japan [27] or as friend in Washington [26], but no concerns are brought up by the users. Kanda et al. [26] attribute the ability of friendship to the robot. But already in an earlier study [27] it is shown, that children lost their interest in the robot after a certain amount of time.

Older People: Scitos [12] interacted with people older than 60. The study investigates the idea of a robot as companion. The only hint towards ethical concerns is mentioned as an idea for future work: "Will there be effects of habituation 


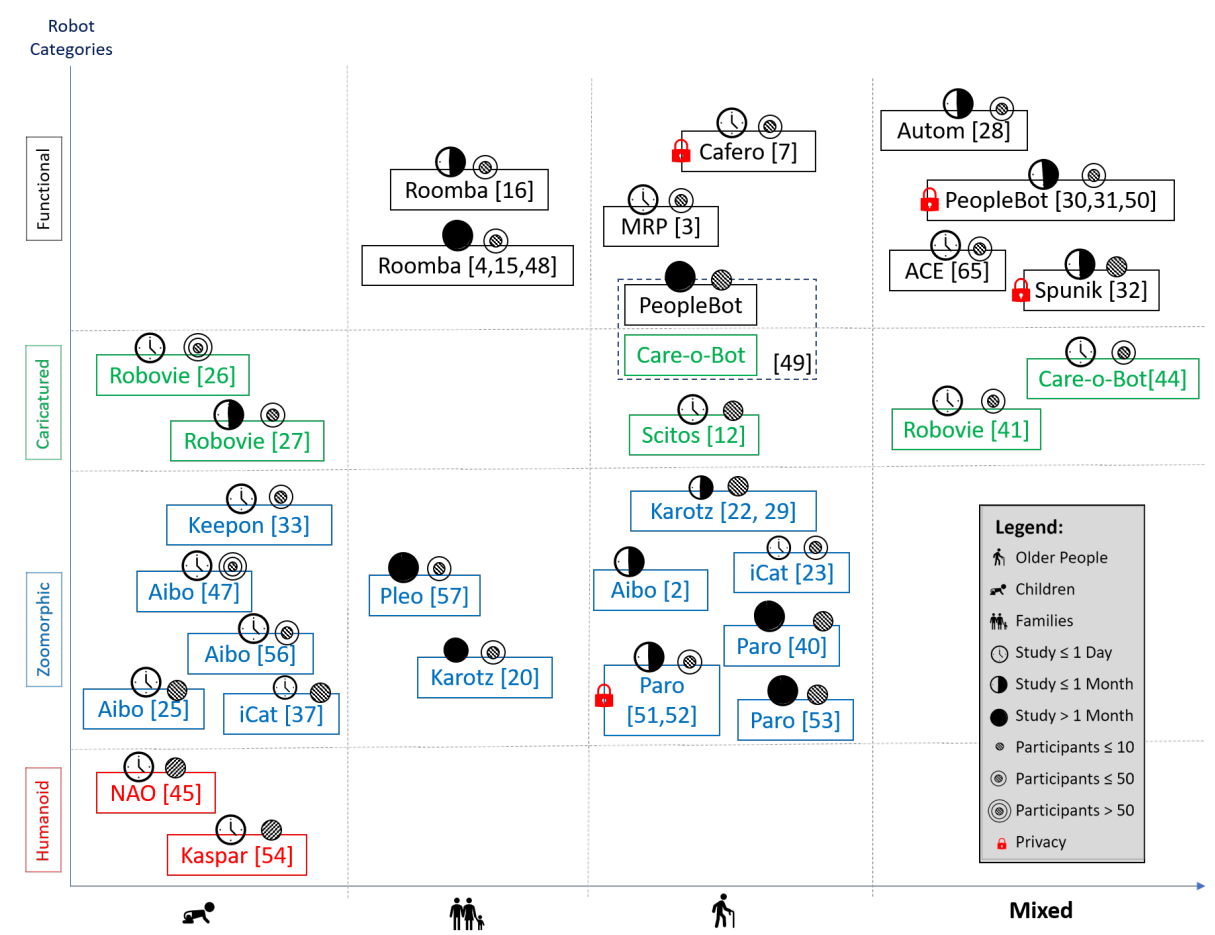

Fig. 5. Overview of Robot Studies

in long-term-use positive (e.g. increasing safety and trust) or negative (e.g. decreasing interest in interaction)". The study of PeopleBot and Care-o-Bot [49] is also part of this category.

Mixed: RoboVie was investigated in the context of shopping mall by Kanda [41]. There it could be used as a shopping assistant for everyone in Japan for three years. As an interesting result most of the adults only tried to interact with the robot when their children wanted to make use of RoboVie. Also Careo-Bot assisted in an electronic market where it should show customers the way to products they asked for [44].

\subsection{Zoomorphic}

Children: Aibo is used in different studies on children. Kahn et al. [25] tested the impact on children in preschool, Weiss et al. [56] evaluated "first time reactions in HRI" in a shopping mall and Stanton et al. [47] tried to support autistic children. All the interventions last less than one hour per interaction and no privacy aspects are stated. Weiss et al. [56] point out the goal of "create[ing] an awareness for robots in general". iCat [37] interacts with children during a chess game. The robot is able the show facial expressions and has a camera in its nose. 
Although it is a study investigating children no ethical aspects are mentioned. Kozima et al. introduced a robot called Keepon [33]. Equipped with a camera and a microphone it is able to react on childrens behavior in a non-verbal way.

Families: Another toy robot is Pleo [57], a dinosaur. It has a camera and two microphones. Six families lived with Pleo from two up to ten month. Important statements of users were "no long-term interest" to use the robot and "with another kind of toy, it may not have been a problem to claim that it sees, but several parents in this study seemed to take such information more literally as technical features.". Parents expressed concerns about the cameras, instead of just accepting them as eyes of the robot. Another study employed the rabbit robot Karotz [20]. Karotz has a camera, a microphone and is able to connect to the internet although the internet connection is not really used.

Older People: Paro is a small seal robot with tactile and noise sensors. Wada et al. $[51,52]$ investigate how older people with dementia react on that robot. Even though Paro has limited functionalities, one old lady prefers playing with Paro rather than with other habitants of the retirement house. Additionally, the psychological effects [40] and the general effects in care with older people of using Paro were investigated without significant results [53]. In 2010 a series of studies with the Karotz started in the Netherlands. Two studies are conducted with people older than fifty $[29,22]$ to get an overview of how older people use robots. Ethical aspects are not mentioned, but users did not like to interact with the robot, because of the lack of functionality. A study with Aibo investigated the differences in the interaction with a robot and a real dog [2]. The robot iCat was used to investigate the acceptance of robots as companions with 30 participants (22 female, 8 male) [23].

\subsection{Anthropomorphic}

Children: Kaspar, a robot with cameras in the form of eyes, played a game with six children under ten years with "low-level socially communicative behaviors" [54]. The session lasts less than one hour. Again no ethical aspects are discussed. Another study with one autistic child was made with a NAO robot [45] for a session of 14 minutes. The robot has four directional microphones, loudspeakers, two cameras and can connect to the Internet autonomously. Concerning ethical aspects it is said that "[...] proven reliability and safety need to be gained in advance" and "safeguarding the wellbeing [...] is crucial to ensure that their rights are always protected", but without any further explanation.

\section{Privacy and Robotics}

In this section the perception of privacy in studies about social robots is discussed and contextualized in the categories of types of robots and user groups.

Robot Groups Functional: A lot of studies are conducted in a public space. Users have typically not much time to interact with a robot. Mainly during 
studies in a home context, ethical concerns are identified. People are changing their habits, even though the robot being in their home has no sensors as cameras or microphones. The reason may be the robots functional appearance. Roomba is considered as a device that can move independently and users do not have control over it [15].

Caricatured: RoboVie and Care-o-Bot were used during several studies in different areas. But for the variety of functionalities there are only very limited examples of real use. Many of the available functionalities are not used or problematic [44]. The Care-o-Bot was e.g. of limited use in an electronic market and because of interfering noise. Additionally, a laboratory study was conducted. Users described the robot as interesting, but not very useful. Cafero was designed explicitly for the requirements of users but the study does not give any hints about the user perception. Instead the researchers list general issues of robotic studies as risks, quality of life (QoL), acceptance or confidentiality [7].

Zoomorphic: A lot of robots are already developed in this area. In the study with Pleo [57] the participants treated it as a real animal and were disappointed of restricted functionalities. Despite the fact, that Karotz has a microphone and a camera, its functionalities are very limited. However, Paro is an already accepted, commercially available robot in elder care. Regardless of the minimal set of functionalities, it is able to react and react to peoples actions in a satisfying way. Apart from Paro, there are not many zoomorphic robots that are accepted or can be used satisfactorily, independent of the user group.

Anthropomorphic: This category of robots is recently developed. Because of limited availability and the costs user interaction in studies is very restricted and it is not possible to investigate this area.

User Groups In several studies, users point out their concerns of using robots. Either they do not want to use a robot at all because of privacy concerns regarding data collection and the loss of private space. Or the users are afraid of using a robot because of a lack of technical expertise. Some users accept the robot in their household but change their behavior because of the fear of surveillance. Robot engineers often state that privacy is an important aspect in robotics but there is no typically further information how concerns should be addressed and how privacy might be achieved. Koay et al. [31] state that participants were glad, that the robot learns helpful information for improving functionality, but in a related study participants expressed their concerns about sensitive information [50].

Children: Interestingly, in studies on children ethical concerns are rarely considered. It is pointed out that privacy is important and respected, but concerns expressed by parents are not described. Children do not have the same perspective as adults and they have a more intuitive way of interacting with technology. Additionally, it is stated that children loose interest in robots after a certain time.

Families: Until now, not many studies were conducted in family homes, because robots are not sufficiently robust, as stated by Graaf et al.[20]. The Roomba 
studies also incorporate families. In the case of Pleo, parents had concerns about accepting cameras as eyes [57].

Older People: Since elder care is one of the two main areas in social robotics, many studies are available. Although the studies do not directly focus on privacy, many users express concerns about various aspects of the area. Especially older people seem to be more sensitive with respect to robots. In the study of Graaf et al. [22] users were able to decide by clicking a button whether they agree to be filmed but it was not transparent if the robot is recording or not.

\section{Discussion}

Pillohealth ${ }^{4}$ realizes personalized medication with the help of Pillo via face recognition. It promotes a secure storage of the medication and an additional mobile application is provided. But what happens in case of errors of the face recognition procedure? What happens, if attackers can manipulate Pillo which is connected to the internet? These questions should not only be raised for Pillo, but for all robots in a social context.

Currently, there are not a lot of robots commercially available [8]. Paro, Roomba and lawnmowers are mainly the only robots on the market. An important result of the studies is that a lot of users are not convinced and satisfied of using robots so far. Researchers still have to face a lot of problems as speech and image recognition and restricted functionalities. Additionally, participants stated a lot of concerns regarding sensors like cameras or microphones, storage of personal data or autonomous behavior. In most of the cases, clearly defined ethical principles are missing. Even though some robot features are not as sensitive as medication, the ethical consequences of every single feature need to be investigated. It is important for users, to be aware of the privacy risks of robots.

Therefore more transparency and a thorough consideration of privacy issues is essential to raise user acceptance. To figure out which aspects need to be dealt with, the features of robots need to be investigated. As already proposed in the questionnaire 1, sensors and actors should be considered because of the inherent risk. By using such a questionnaire, users might be able to understand the risks and possible countermeasures. Because the questionnaire has a focus on network security, it is important to address privacy aspects in addition. The model of seven types of privacy is a general approach, to investigate ethical aspects with a focus on privacy [17]. The following Table 2 gives an overview based on typical sensors employed in robotics. Depending on sensors and accompanying functionalities, different types of privacy are violated, e.g. with a camera, a robot is able to track people and their environment. This may influence users in their behavior [15]. Especially in the care area, this must be clearly transparent [22]. Concerning these aspects further correlation to other smart devices need to be investigated.

Another important privacy issue is the fact of Thoughts and Feelings. Robots are described as a friend or companion in many of the studies. That implies blind

\footnotetext{
${ }^{4}$ http://pillohealth.com/
} 


\begin{tabular}{|c|c|c|c|c|c|c|}
\hline $\begin{array}{c}\text { Privacy of } \\
\ldots\end{array}$ & Camera & GPS & $\begin{array}{c}\text { Loud- } \\
\text { speaker }\end{array}$ & $\begin{array}{l}\text { Micro- } \\
\text { phones }\end{array}$ & $\begin{array}{l}\text { Tactile } \\
\text { Sensors }\end{array}$ & $\mathrm{WiFi}$ \\
\hline \multicolumn{7}{|l|}{ the Person } \\
\hline $\begin{array}{c}\text { Behavior } \\
\text { and Action }\end{array}$ & $\mathrm{X}$ & $\mathrm{X}$ & $\mathrm{X}$ & $\mathrm{X}$ & $\mathrm{X}$ & \\
\hline $\begin{array}{c}\text { Communi- } \\
\text { cation }\end{array}$ & & & $\mathrm{X}$ & $\mathrm{X}$ & $\mathrm{X}$ & $\mathrm{X}$ \\
\hline $\begin{array}{c}\text { Data and } \\
\text { Image }\end{array}$ & $\mathrm{X}$ & & & $\mathrm{X}$ & & $\mathrm{X}$ \\
\hline $\begin{array}{l}\text { Thought } \\
\text { and } \\
\text { Feeling }\end{array}$ & & & $\mathrm{X}$ & & $\mathrm{X}$ & \\
\hline $\begin{array}{c}\text { Location } \\
\text { and Space }\end{array}$ & $\mathrm{X}$ & $\mathrm{X}$ & & & & \\
\hline Association & $\mathrm{X}$ & & & $\mathrm{X}$ & & $\mathrm{X}$ \\
\hline
\end{tabular}

Table 2. Types of Privacy and the impact of Technical Conditions

trust and an absolutely openness towards them [21]. Through the humanization of human-robot interaction, the topic of privacy gains even more importance. Cynthia Breazeal introduced the relationship between emotional engagement and functional utility $[5,23]$. The more functionality a robot has, the more intimate is the emotional binding. The more functional it looks like, the more it is seen as a machine and not as a companion . Nevertheless, appearance of a robot is not the main characteristic for being skeptical.

In general, robots should not be too complex but more useful and helpful for daily routines. To address privacy concerns, transparency is needed concerning collection of personal data and it is important that the user is able to intervene, e.g. by switching off certain features. A lot of participants criticized the available features and missed some helpful ones. A robot e.g. does not need a camera when the functionalities using the camera are not perceived beneficial. Therefore it is of utter importance to involve potential users in robot design [7,34]. Users should be asked about preferences and aversions. This would ensure whether a specific sensor is really needed or not and thereupon a more specific analysis is possible.

\section{Conclusion}

Summarized, only a few ethical aspects have been investigated in robot studies so far. There are already some doubts mentioned regarding reduction of human contact, loss of control and loss of privacy related to recordings. The concern of emotions \& attachments is not seen as problem so far.

Privacy should be a broad and important topic in robotic research. Zoomorphic robots, preferably used in health care are mostly simplistic but on the other hand very successful. With increasing demands, the complexity of robots will in- 
crease. Cameras, microphones and internet access will be a standard for robots. This allows an enlargement of robot functionalities, especially concerning social conversation and interaction between user and robot. Therefore the parallels to already available intrusive technical devices like mobile phones and their mobile applications are important to investigate. But for robots to be successful on the market, it must be helpful. If the robots have sensors it must be clear, why and for what reasons and scenarios they are needed. The two main target groups for social robots children and older people (with physical/psychological deficits) might have difficulties to be aware of the risks using them and the high complexity. Therefore risks and requirements need to be investigated during the whole development process to get a more transparent view.

Acknowledgment This work was supported by the Ministry for Science and Culture of Lower Saxony as part of the program "Gendered Configurations of Humans and Machines (KoMMa.G)".

\section{References}

1. Amirfar, V.A.: A little robot with big promise may be future of personalized health care. Pharmacy Today 22(9), 38 (September 2016)

2. Banks, M.R., Willoughby, L.M., Banks, W.A.: Animal-assisted therapy and loneliness in nursing homes: use of robotic versus living dogs. Journal of the American Medical Directors Association 9(3), 173-177 (2008)

3. Beer, J.M., Takayama, L.: Mobile remote presence systems for older adults: acceptance, benefits, and concerns. In: Proceedings of the 6th international conference on Human-robot interaction. pp. 19-26. ACM (2011)

4. Breazeal, C.: How Robotic Products Become Social Products: An Ethnographic Study of Cleaning in the House. ACM, New York, NY (2007), http://dl.acm.org/citation.cfm?id=1228716

5. Breazeal, C.: A social robot in every home. MIT Media Lab (May 2015)

6. Breazeal, C.L.: Designing sociable robots (intelligent robotics and autonomous agents). MIT Press, Cambridge (2002)

7. Broadbent, E., Jayawardena, C., Kerse, N., et al.: Human-robot interaction research to improve quality of life in elder care - an approach and issues. In: AAAI Workshop Conference on Artificial Intelligence (2011)

8. Broadbent, E., Stafford, R., MacDonald, B.: Acceptance of healthcare robots for the older population: review and future directions. International Journal of Social Robotics 1(4), 319-330 (2009)

9. Coeckelbergh, M., Pop, C., Simut, R., Peca, A., Pintea, S., David, D., Vanderborght, B.: A survey of expectations about the role of robots in robot-assisted therapy for children with ASD: Ethical acceptability, trust, sociability, appearance, and attachment. Science and engineering ethics 22(1), 47-65 (2016)

10. Denning, T., Matuszek, C., Koscher, K., Smith, J.R., Kohno, T.: A spotlight on security and privacy risks with future household robots: attacks and lessons. In: Proceedings of the 11th international conference on Ubiquitous computing. pp. 105-114. ACM (2009) 
11. DiSalvo, C.F., Gemperle, F., Forlizzi, J., Kiesler, S.: All robots are not created equal: the design and perception of humanoid robot heads. In: Proceedings of the 4th conference on Designing interactive systems: processes, practices, methods, and techniques. pp. 321-326. ACM (2002)

12. Döring, N., Richter, K., Gross, H.M., Schröter, C., Mueller, S., Volkhardt, M., Scheidig, A., Debes, K.: Robotic companions for older people: A case study in the wild. Studies Health Technol Inform 2015b 219, 147-152 (2016)

13. Duffy, B.R.: Anthropomorphism and the social robot. Robotics and autonomous systems 42(3), 177-190 (2003)

14. Feil-Seifer, D., Mataric, M.: Socially assistive robotics. IEEE Robotics \& Automation Magazine 18(1), 24-31 (2011)

15. Fink, J., Bauwens, V., Kaplan, F., Dillenbourg, P.: Living with a vacuum cleaning robot. International Journal of Social Robotics 5(3), 389-408 (2013)

16. Fink, J., Bauwens, V., Mubin, O., Kaplan, F., Dillenbourg, P.: People's perception of domestic service robots: Same household, same opinion? In: Proceedings of the 3rd International Conference on Social Robotics, ICSR 2011. pp. 204-213. No. EPFL-CONF-170409, Springer (2011)

17. Finn, R.L., Wright, D., Friedewald, M.: Seven types of privacy. In: European data protection: coming of age, pp. 3-32. Springer (2013)

18. Fong, T., Nourbakhsh, I., Dautenhahn, K.: A survey of socially interactive robots. Robotics and autonomous systems 42(3), 143-166 (2003)

19. de Graaf, M.M.A., Ben Allouch, S., van Dijk, J.A.G.M.: What makes robots social? a user's perspective on characteristics for social human-robot interaction. In: Tapus, A., André, E., Martin, J.C., Ferland, F., Ammi, M. (eds.) Social Robotics, Lecture Notes in Computer Science, vol. 9388, pp. 184-193. Springer International Publishing, Cham (2015)

20. de Graaf, M., Ben Allouch, S., van Dijk, J.: Why do they refuse to use my robot? In: Mutlu, B., Tscheligi, M., Weiss, A., Young, J.E. (eds.) Proceedings of the 2017 ACM/IEEE International Conference on Human-Robot Interaction - HRI '17. pp. 224-233. ACM Press, New York, New York, USA (2017)

21. de Graaf, M.M.: An ethical evaluation of human-robot relationships. International journal of social robotics 8(4), 589-598 (2016)

22. de Graaf, M.M., Allouch, S.B., Klamer, T.: Sharing a life with harvey: Exploring the acceptance of and relationship-building with a social robot. Computers in Human Behavior 43, 1-14 (2015)

23. Heerink, M., Kröse, B., Evers, V., Wielinga, B., et al.: The influence of social presence on acceptance of a companion robot by older people. Journal of Physical Agents 2(2), 33-40 (2008)

24. International Federation of Robotics: Executive summary world robotics 2016 service robots, https://ifr.org/downloads/press/02_2016/Executive_Summary_Service_Robots 2016.pdf

25. Kahn, P.H., Friedman, B., Perez-Granados, D.R., Freier, N.G.: Robotic pets in the lives of preschool children. Interaction Studies 7(3), 405-436 (2006)

26. Kahn Jr, P.H., Kanda, T., Ishiguro, H., Freier, N.G., Severson, R.L., Gill, B.T., Ruckert, J.H., Shen, S.: robovie, you'll have to go into the closet now: Children's social and moral relationships with a humanoid robot. Developmental psychology 48(2), $303(2012)$

27. Kanda, T., Hirano, T., Eaton, D., Ishiguro, H.: Interactive robots as social partners and peer tutors for children: A field trial. Human-computer interaction 19(1), 61-84 (2004) 
28. Kidd, C.D., Breazeal, C.: Robots at home: Understanding long-term human-robot interaction. In: Intelligent Robots and Systems, 2008. IROS 2008. IEEE/RSJ International Conference on. pp. 3230-3235. IEEE (2008)

29. Klamer, T., Ben Allouch, S., Heylen, D.: adventures of harvey-use, acceptance of and relationship building with a social robot in a domestic environment. HumanRobot Personal Relationships pp. 74-82 (2011)

30. Koay, K.L., Syrdal, D.S., Walters, M.L., Dautenhahn, K.: Living with robots: Investigating the habituation effect in participants' preferences during a longitudinal human-robot interaction study. In: Robot and Human interactive Communication, 2007. RO-MAN 2007. The 16th IEEE International Symposium on. pp. 564-569. IEEE (2007)

31. Koay, K.L., Syrdal, D.S., Walters, M.L., Dautenhahn, K.: Five weeks in the robot house-exploratory human-robot interaction trials in a domestic setting. In: Advances in Computer-Human Interactions, 2009. ACHI'09. Second International Conferences on. pp. 219-226. IEEE (2009)

32. Koay, K., Syrdal, D., Dautenhahn, K., Arent, K., Małek, Ł., Kreczmer, B.: Companion migration-initial participants feedback from a video-based prototyping study. Mixed Reality and Human-Robot Interaction pp. 133-151 (2011)

33. Kozima, H., Michalowski, M.P., Nakagawa, C.: Keepon. International Journal of Social Robotics 1(1), 3-18 (2009)

34. Lee, H.R., Šabanović, S., Chang, W.L., Nagata, S., Piatt, J., Bennett, C., Hakken, D.: Steps toward participatory design of social robots: Mutual learning with older adults with depression. In: Proceedings of the 2017 ACM/IEEE International Conference on Human-Robot Interaction. pp. 244-253. ACM (2017)

35. Lee, K.M., Peng, W., Jin, S.A., Yan, C.: Can robots manifest personality? an empirical test of personality recognition, social responses, and social presence in human?robot interaction. Journal of Communication 56(4), 754-772 (2006)

36. Leite, I., Martinho, C., Paiva, A.: Social robots for long-term interaction: a survey. International Journal of Social Robotics 5(2), 291-308 (2013)

37. Leite, I., Martinho, C., Pereira, A., Paiva, A.: As time goes by: Long-term evaluation of social presence in robotic companions. In: Robot and Human Interactive Communication, 2009. RO-MAN 2009. The 18th IEEE International Symposium on. pp. 669-674. IEEE (2009)

38. Murphy, R., Woods, D.D.: Beyond asimov: the three laws of responsible robotics. IEEE Intelligent Systems 24(4) (2009)

39. Pineau, J., Montemerlo, M., Pollack, M., Roy, N., Thrun, S.: Towards robotic assistants in nursing homes: Challenges and results. Robotics and autonomous systems 42(3), 271-281 (2003)

40. Robinson, H., MacDonald, B., Kerse, N., Broadbent, E.: The psychosocial effects of a companion robot: a randomized controlled trial. Journal of the American Medical Directors Association 14(9), 661-667 (2013)

41. Sabelli, A.M., Kanda, T.: Robovie as a mascot: A qualitative study for long-term presence of robots in a shopping mall. IJ Social Robotics 8(2), 211-221 (2016)

42. Scassellati, B.M.: Foundations for a Theory of Mind for a Humanoid Robot. Ph.D. thesis, Massachusetts Institute of Technology (2001)

43. Scheeff, M., Pinto, J., Rahardja, K., Snibbe, S., Tow, R.: Experiences with sparky, a social robot. In: Socially Intelligent Agents, pp. 173-180. Springer (2002)

44. Schmitt, C., Schäfer, J., Burmester, M.: Wie wirkt der care-o-bot 4 im verkaufsraum? Mensch und Computer 2017-Usability Professionals (2017) 
45. Shamsuddin, S., Yussof, H., Ismail, L.I., Mohamed, S., Hanapiah, F.A., Zahari, N.I.: Initial response in hri- a case study on evaluation of child with autism spectrum disorders interacting with a humanoid robot nao. Procedia Engineering 41, 1448-1455 (2012)

46. Sharkey, A., Sharkey, N.: Granny and the robots: Ethical issues in robot care for the elderly. Ethics and Information Technology 14(1), 27-40 (2010)

47. Stanton, C.M., Kahn, P.H., Severson, R.L., Ruckert, J.H., Gill, B.T.: Robotic animals might aid in the social development of children with autism. In: Human-Robot Interaction (HRI), 2008 3rd ACM/IEEE International Conference on. pp. 271-278. IEEE (2008)

48. Sung, J., Christensen, H.I., Grinter, R.E.: Robots in the wild: understanding longterm use. In: Proceedings of the 4th ACM/IEEE international conference on $\mathrm{Hu}-$ man robot interaction. pp. 45-52. ACM (2009)

49. Syrdal, D.S., Dautenhahn, K., Koay, K.L., Ho, W.C.: Views from within a narrative: Evaluating long-term human-robot interaction in a naturalistic environment using open-ended scenarios. Cognitive computation 6(4), 741-759 (2014)

50. Syrdal, D.S., Walters, M.L., Otero, N., Koay, K.L., Dautenhahn, K.: He knows when you are sleeping-privacy and the personal robot companion. In: Proc. workshop human implications of human-robot interaction, association for the advancement of artificial intelligence (AAAI07). pp. 28-33 (2007)

51. Wada, K., Shibata, T.: Living with seal robots - its sociopsychological and physiological influences on the elderly at a care house. IEEE Transactions on Robotics 23(5), 972-980 (2007)

52. Wada, K., Shibata, T., Kawaguchi, Y.: Long-term robot therapy in a health service facility for the aged-a case study for 5 years. In: Rehabilitation Robotics, 2009. ICORR 2009. IEEE International Conference on. pp. 930-933. IEEE (2009)

53. Wagemaker, E., Dekkers, T.J., Agelink van Rentergem, J.A., Volkers, K.M., Huizenga, H.M.: Advances in mental health care: Five $n=1$ studies on the effects of the robot seal paro in adults with severe intellectual disabilities. Journal of Mental Health Research in Intellectual Disabilities pp. 1-12 (2017)

54. Wainer, J., Dautenhahn, K., Robins, B., Amirabdollahian, F.: A pilot study with a novel setup for collaborative play of the humanoid robot kaspar with children with autism. International Journal of Social Robotics 6(1), 45-65 (2014)

55. Weiss, A., Igelsböck, J., Tscheligi, M., Bauer, A., Kühnlenz, K., Wollherr, D., Buss, M.: Robots asking for directions: the willingness of passers-by to support robots. In: Proceedings of the 5th ACM/IEEE international conference on Human-robot interaction. pp. 23-30. IEEE Press (2010)

56. Weiss, A., Wurhofer, D., Tscheligi, M.: i love this dogchildrens emotional attachment to the robotic dog aibo. International Journal of Social Robotics 1(3), 243-248 (2009)

57. Ylva Fernaeus, Maria Hkansson, M.J.S.L.: How do you Play with a Robotic Toy Animal? A long-term study of Pleo. ACM, New York, NY (2010), http://dl.acm.org/citation.cfm?id=1810543 